1

2

3

\title{
Energy dissipation of pulsating bubbles in compressible fluids using the Eulerian finite-element method
}

\author{
Zhao-Li Tian ${ }^{\mathrm{a}, \mathrm{c}}$, Yun-Long Liu ${ }^{\mathrm{a}, \mathrm{c}, *}$, A-Man Zhang, ${ }^{\mathrm{a}, *}$, Longbin Tao $^{\mathrm{b}}$ \\ ${ }^{a}$ College of Shipbuilding Engineering, Harbin Engineering University, Harbin 150001, China \\ ${ }^{b}$ Department of Naval Architecture, Ocean 83 Marine Engineering, University of Strathclyde, \\ Glasgow, United Kingdom \\ ${ }^{c}$ Division of Applied Mathematics, Brown University, Providence RI 02912, USA
}

\begin{abstract}
The energy dissipation mechanisms of bubble pulsation in compressible fluids have always been a significant aspect of research into bubble dynamics. In this paper, bubble dynamics in compressible fluids are investigated numerically with the Eulerian finite-element method (EFEM), and the energy dissipation due to the wave effects of the compressible surrounding fluid is analyzed. The present model is validated by comparing with experimental results. Results from both the simulation and experiment show that bubble fragmentation also contributes to the energy dissipation, which has seldom been discussed before. It is also shown that the initial discontinuity is significant to the energy dissipation which is non-trivial to simulate in 1-dimensional bubble dynamics equations like the Gilmore equation. Then, the relationship between dissipated energy and bubble maximum radii in adjacent pulsating cycles is formulated to quantitatively evaluate the energy dissipation during a pulsating cycle. At last, based on the linearized theory of the energy conservation of the bubble system, a new non-dimensional parameter $M_{a}$ is modified from the Mach number to represent the energy dissipation due to wave effects. With simulation and discussion on cases with different initial pressure and sound speed, it is found that
\end{abstract}

\footnotetext{
*Corresponding author

** Corresponding author

Email addresses: yunlong_liu@hrbeu.edu.cn (Yun-Long Liu), zhangaman@hrbeu.edu.cn (A-Man Zhang)
} 
the dissipated energy is related linearly to $M_{a}$, which can be used to predict the energy dissipation of a new case.

Keywords: Bubble dynamics, Compressible fluid, Eulerian finite-element method (EFEM), Energy dissipation

\section{Introduction}

Pulsating bubbles initially studied by Rayleigh [1] have wide applications, such as ice breaking by collapsing bubble[2], underwater explosion (UNDEX) bubbles that threatened nearby warships[3-7], air-gun bubbles used to explore ocean resources[810], and vapor bubbles used in ink printers[11, 12]. If the bubbles are placed in water with a high-pressure internal gas produced by an explosive detonation or some other form of energy release, they then expand because of the pressure difference across the bubble interface and start pulsating. Under the effects of pressure gradients or boundaries such as solid walls or free surfaces in the surrounding fluid, the bubbles will exhibit complex nonspherical dynamics, such as jet development or fragmentation $[6,13-17]$.

When only the first pulsation cycle is considered in the evolution of a bubble, the surrounding fluid is commonly assumed to be incompressible to simplify the theoretical or numerical model, such as with the boundary element method (BEM) $[6$, 13, 18-22] and the finite volume method (FVM)[23]. With these approaches, there is no energy dissipation through sound radiation. Thus, the simulated maximum radius of each pulsating cycle keeps constant, as a result of the conservativeness of energy when the bubble moves spherically[24-26], which violates the fact that the maximum radii decay quickly observed in experiments. For example, the maximum radius of the second pulsation cycle of an underwater explosion bubble can be as small as only $50 \%$ of the first one[24, 27]. Given that the compressibility of the surrounding fluid is one of the essential energy dissipation mechanisms[24, 28], it must be included when the multi-cycle pulsation process is concerned.

Bubble dynamics in weakly compressible fluids have been studied extensively for both spherical and nonspherical bubbles. The equation of state (EoS) of the 
fluid is usually linearized for simplification. For spherical bubble dynamics, the linearized wave equation[29], matched asymptotic expansion method[30, 31], and double asymptotic approximation (DAA) method[24, 32] are used to describe the weakly compressible behavior of the surrounding flow. It has been found that compressibility can lead to a significant reduction in the maximum radius in a sequence of pulsation cycles.

Recently, the bubble dynamic research has expanded to include nonspherical bubble motion. Wang [27, 28, 33] employed matches between the Laplace's equation for the inner flow and the linear wave equation for the outer flow to establish a secondorder bubble dynamics model considering the weak compressibility of the surrounding flow. Zhang et al. [25] established a similar three-dimensional bubble dynamics model by unifying the prophase approximations controlled by the linear wave equation and the anaphase approximation controlled by the retarded potential integral equation. Those studies took into account the compressibility of the surrounding fluid but ignored the wave effect of the internal gas and assumed that its pressure was uniform. Xiao et al. [26] expanded the inner field DAA method from one-dimensional to threedimensional problems and investigated further the influences of the wave effects of the internal gas and the outer fluid. These numerical approaches based on the BEM can predict the energy dissipation of an oscillating bubble accurately compared with experimental results. However, there are still several difficulties. Firstly, linear and weak compressibility assumptions are used so that the propagation of a shock wave and the attached energy dissipation have to be ignored; Secondly, the determination of initial conditions is nontrivial. For example, Wang [28] determined the initial conditions by a backward integration of the Keller equation but not by a physically meaningful way. At last, bubble fragmentation is nontrivial with BEM. The difficulty is not only from topology changes of boundary mesh, but also arises from the singleconnected assumption on which the potential theory is based. Because each time a fragment bubble splits off, a new vortex ring may be required to cancel the induced circulation of the velocity potential[14].

Benefiting from the dramatically development of the computing power, the numerical methods using the spatial domain mesh are increasingly employed in simu- 

Eulerian finite-element method(EFEM)[36, 37], the spectral element marker particle $\operatorname{method}[38]$ and the free Lagrange method[39]. These methods can easily treat the compressibility of the fluid using proper EoSs and do not suffer from the difficulties in the numerical treatment when the topology of the fluid interface changes in BEM. The EFEM was first established by Noh [40]. Later, Benson [41] systematically reviewed the computational methods in Eulerian hydrocodes and presented the main framework of EFEM, which separates the calculation of the Euler equations into two phases. During one time increment, a Lagrangian phase is implemented first, and the mesh is advanced with the material, which is usually solved using the finite-element method (FEM). The mesh is then moved back to its original position, and the transport of variables between elements is considered at the end of the increment, which is referred to as the Eulerian phase and solved with the upwind schemes developed for Eulerian formulations. Benson [42] developed EFEM further by proposing a new convection method for node-centered variables such as momentum. The EFEM has been successfully applied in the simulation of bubble dynamics by Liu et al. [36] and Tian et al. [37]. With the features of fully considered compressibility, physically meaningful initial conditions and bubble fragmentation without special treatment, the difficulties faced by the BEM do not exist anymore.

In the present paper, the multi-phase EFM is reformulated based on Liu et al. [36] with which nonspherical pulsating bubble dynamics in compressible free field is simulated to investigate the energy dissipation mechanisms considering nonlinear compressibility of surrounding fluid. Using a linearized theoretical method, a new non-dimensional parameter $M_{a}$ is introduced to examine the energy dissipation of a pulsating bubble in a compressible fluid through numerical and theoretical analysis.

This paper is organized as follows. In Section 2, theoretical and numerical formulations are presented for the axisymmetric model of bubble dynamics in a compressible fluid. A benchmark case is then simulated in Section 2.7 and compared with experimental results to validate the model and analyze the energy dissipation mechanism of the pulsating bubble in the free field. In Section 4, how compressibility affects energy dissipation and bubble evolution is studied through theoretical analysis 
and simulation results for several non-dimensional cases. Finally, some conclusions are drawn.

\section{Theoretical and numerical models}

\subsection{Problem configuration and assumptions}

To formulate the problem, the configuration and basic assumptions are firstly presented here. A bubble is initially placed in still water with highly compressed internal gaseous contents which might be explosion products for UNDEX bubbles or compressed air for air-gun bubbles. The bubble then begins to expand because of the pressure difference and is taken to be sufficiently far from the flow boundaries (e.g., free surface, solid bottom) that the surrounding flow can be treated as infinite. Thus, a cylindrical coordinate system is established, where $r, \theta$, and $z$ are the radial, angular, and axial coordinates, respectively. The origin is at the center of the bubble and the $z$ axis points upward, as shown in Fig. 1. Ignoring the surface instabilities that may destroy the axisymmetry of the problem, the mathematical model is simplified to be independent of the angular coordinate $\theta$. Thus, the following discussions are based on the axisymmetric assumption that has been widely used in previous studies on bubble dynamics[12, 13, 20, 27, 28, 33, 37, 43]. The computational domain is taken as a rectangle of size $a \times 2 a$.

Following previous studies of bubble dynamics, surface tension, viscosity, and thermal conduction are all neglected[4, 6, 23, 33]. The stress tensor can be expressed simply as the inverse of the scalar pressure $p$. In the present cylindrical coordinate system, the Euler equations describing the motion of the fluid field read

$$
\left\{\begin{array}{l}
\rho_{t}+\nabla \cdot(\rho \mathbf{u})+\rho \frac{u}{r}=0 \\
(\rho u)_{t}+\nabla \cdot(\rho u \mathbf{u})+p_{r}+\rho \frac{u^{2}}{r}=0 \\
(\rho v)_{t}+\nabla \cdot(\rho v \mathbf{u})+p_{z}+\rho \frac{u v}{r}=-\rho g \\
\left(\rho e_{i n}\right)_{t}+\nabla \cdot\left(\rho e_{i n} \mathbf{u}\right)+p\left(\nabla \cdot \mathbf{u}+\frac{u}{r}\right)+\rho e_{i n} \frac{u}{r}=0
\end{array}\right.
$$

which stand for the mass, momentum in $r$ and $z$ directions, and the internal energy 
conservation, respectively. Here, $\mathbf{u}=(u, v)^{T}$ is the column vector of velocity, $\rho$ is the density of the fluid, $u$ and $v$ are the velocity in the directions of $r$ and $z, g$ is the gravity acceleration and $e_{i n}$ represents the specific internal energy. The subscripts $t$, $r$ and $z$ indicate the partial derivative about the corresponding variables. Besides, $\nabla=\left(\frac{\partial}{\partial r}, \frac{\partial}{\partial z}\right)$ is the gradient operator.

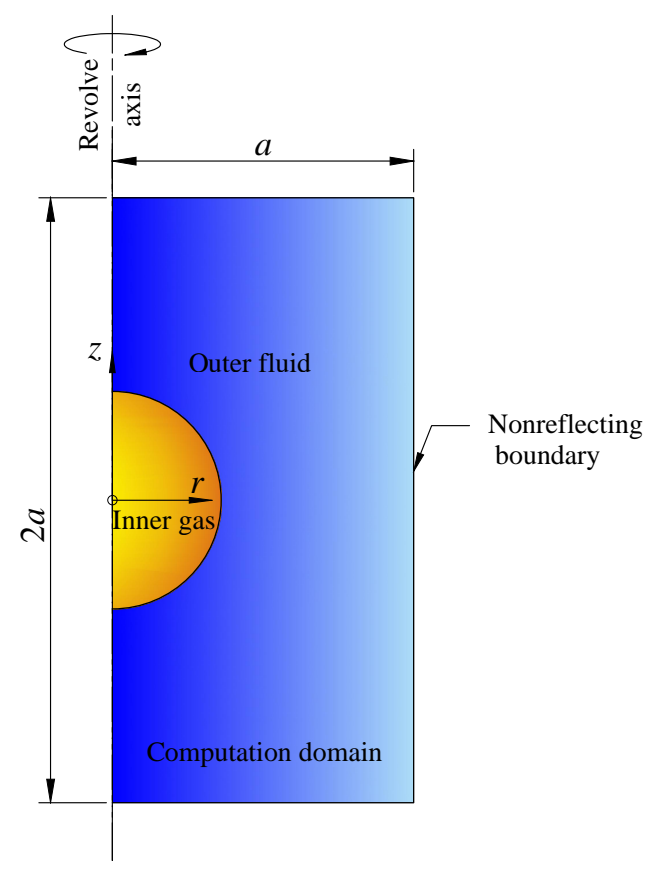

Figure 1: Configuration of pulsating bubble in compressible fluid.

\subsection{Initial and boundary conditions}

As stated in Sect.2.1, the bubble is initially placed in the still water. Thus, except for the initial radius of the bubble, only the parameters related to the initial state of the internal gas need to be initialized herein. In this paper, they are determined by two different methods according to the EoSs chosen for the internal gas of the bubble as shown in Sect.2.5.2. When the internal gas is modeled with the Jones-WilkensLee (JWL) EoS for UNDEX bubble, the initial radius $R_{0}$, specific internal energy $e_{i n 0}$, and density of the internal gas $\rho_{0}$ are taken as those of the explosive as shown in Tab.2. This straightforward method is physically meaningful and used in Sect.2.7 
for the case involving actual UNDEX. Otherwise, the ideal gas EoS is used where the initial parameters must be chosen artificially. It has been shown by Klaseboer et al. [4] that the initial pressure $P_{0}$ and the radius $R_{0}$ must satisfy the following relationship so that the bubble can expand to the specific maximum radius $R_{m}$ in incompressible fluid

$$
P_{\infty}\left(R_{m}^{3}-R_{0}^{3}\right)(1-\gamma)=P_{0} R_{0}^{3 \gamma}\left(R_{m}^{-3 \gamma+3}-R_{0}^{-3 \gamma+3}\right),
$$

which is derived from the fact that the surrounding flow dose zeros work on the bubble from the beginning of the expansion to the first time the bubble reaches its maximum radius. Here, $P_{\infty}=P_{a t m}+\rho_{f} g h$ is the ambient pressure at the bubble center with $P_{a t m}, \rho_{f}$ and $h$ representing the atmosphere, the density of the surrounding fluid and the depth of the bubble; $\gamma$ is the ratio of specific heat of the internal gas. Thus, if either $P_{0}$ or $R_{0}$ is known, the other one can be calculated by solving the nonlinear equation above. In this paper, $P_{0}$ will be given and $R_{0}$ will be calculated correspondingly. If the surrounding fluid is compressible, the maximum radius of the pulsating bubble can not reach the given $R_{m}$ because of the energy dissipation effect. Thus, the actual maximum radius in the $i$ th pulsating cycle that the bubble can reach is denoted by $R_{m}^{(i)}$ to be distinguished from $R_{m}$.

As for the boundary condition for this problem, the only boundary needs to be considered is the outer boundary of the truncated computational domain. Using the linear assumption in far-field at the outer boundary of the computational domain $\Omega$ and assuming that the pressure wave is propagating spherically through the boundary, the non-reflecting boundary condition is given by Liu et al. [36]:

$$
p_{t}(\mathbf{r})=\rho c \mathbf{u}_{t} \cdot \mathbf{e}+\kappa \rho\left(p-p_{0}\right) \text { for } \mathbf{r} \in \partial \Omega,
$$

of which the truncation error is proportional to $a^{-2}$. Here, $a$ denotes the computational domain size, as shown in Fig. 1, $c$ is the local speed sound, e is the unit vector pointing in the direction of pressure wave propagating which is approximated by $\frac{\mathbf{r}-\mathbf{r}_{c}}{\left|\mathbf{r}-\mathbf{r}_{c}\right|}$ and $\kappa=\frac{1}{\left|\mathbf{r}-\mathbf{r}_{c}\right|}$ represents the local curvature of the pressure wave front by 
denoting $\mathbf{r}=(r, z)$ as the position of the non-reflecting boundary and $\mathbf{r}_{c}$ representing the position of the current bubble center.

\subsection{Eulerian finite-element method with operator split}

Herein, EFEM with operator split[36, 40, 41] is adopted to simulate the bubble motion formulated by Eq.(1) and Eq.(3). The main idea is to split the system into two phases. In the first phase, the convection term in Eq.(1) is neglected so that the mesh is flow along with the fluid material in one single time increment. This is done by solving the momentum equations in Eq.(1) with the traditional explicit finiteelement method, and the conservation of mass and energy are computed thereafter with the new results. In the second phase, the convection terms are compensated by moving the deformed mesh back to its original position while the fluid material is fixed. Through the combination of these two steps, the fluid material moves forward continuously with time while the mesh is fixed to maintain the Eulerian grid. The overall numerical procedures of the present model are shown in Fig. 2.

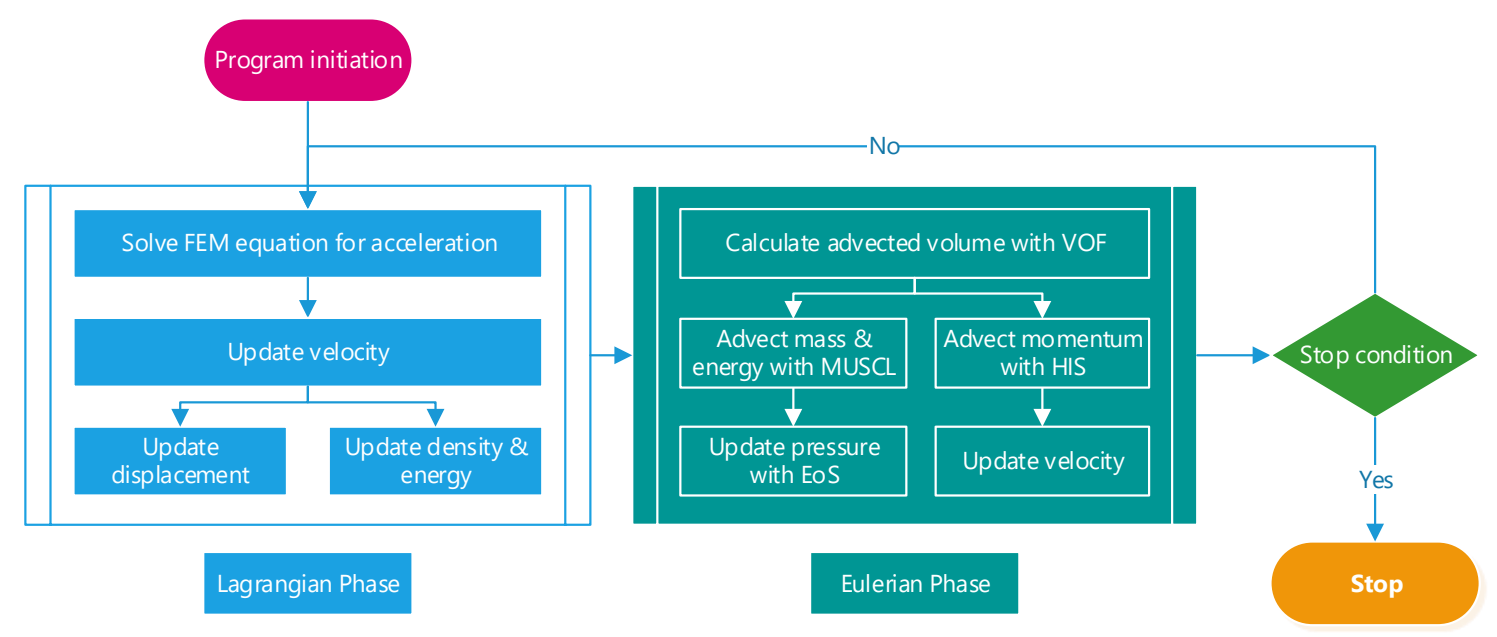

Figure 2: Flow chart of the numerical procedures.

\subsection{Lagrangian phase with explicit FEM}

In this phase, the convection term is dropped so that the reduced Euler equations follow the perspective of Lagrange. In the previous two paper using the EFEM[36, 
37], the momentum equations are formulated by the balance of the forces acting on a slice of fluid in the cylindrical coordinate system. Thus, singularity arises near the rotating axis because of the factor $\frac{1}{r}$. Then, the momentum equations are approximated by the following weak formula with $\phi$ denoting the trail function to avoid the above problem.

$$
\left\{\begin{array}{l}
\frac{d}{d t} \iint_{\Omega} \rho u \phi \mathrm{d} s=\iint_{\Omega} p \phi_{r} \mathrm{~d} s-\int_{\partial \Omega} p \phi n_{r} \mathrm{~d} l \\
\frac{d}{d t} \iint_{\Omega} \rho v \phi \mathrm{d} s=\iint_{\Omega}\left(p \phi_{z}-\rho g \phi\right) \mathrm{d} s-\int_{\partial \Omega} p \phi n_{z} \mathrm{~d} l
\end{array}\right.
$$

where $\Omega$ is the discretized computational domain and $\partial \Omega$ is its outer boundary; $\mathbf{n}=\left(n_{r}, n_{z}\right)$ is the unit normal vector; $\phi_{r}$ and $\phi_{z}$ are the directional derivatives of $\phi$ in directions of $r$ and $z$, respectively.

The multi-phase problem is considered by employing the volume of fluid (VOF) method[36, 44]. The fluid fraction function $f^{(i)}$ is introduced to represent the volume ratio of the $i$ th fluid phase in an element with the limitation of $f^{(i)} \geq 0$ and $\sum_{i=0}^{N_{f}} f^{(i)}=1$, where $N_{f}$ is the number of the fluids. During the Lagrangian phase, the mixture theory is used to solve the momentum equation. i.e., the fluid density in Eq.(4) is expressed as

$$
\rho=\sum_{i=1}^{N_{f}} \rho^{(i)} f^{(i)},
$$

where $N_{f}$ is the number of fluid phases and $\rho^{(i)}$ is the density of the $i$ th fluid phase.

Assuming that the quantities on the right-hand side of Eq. (4) are known, the fluid acceleration $\frac{d u}{d t}$ and $\frac{d v}{d t}$ are calculated by discretizing the computational domain into finite elements. Then, both of the 2 equations in Eq.(4) can be written as the matrix form

$$
\mathbf{M A}_{d}=\mathbf{F}_{d}
$$

where $d$ equals $r$ or $z$ depending on which direction is considered, $\mathbf{M}$ is the mass matrix, $\mathbf{A}_{d}$ is the column vector of the nodal acceleration, and $\mathbf{F}_{d}$ is the vector of 
the equivalent nodal force. Their components can be expressed as

$$
\begin{gathered}
M_{P Q}=\iint_{\Omega} \rho \phi^{P} \phi^{Q} \mathrm{~d} s \\
F_{P}=\left\{\begin{array}{l}
\iint_{\Omega} p \phi_{r}^{P} \mathrm{~d} s-\int_{\partial \Omega} p \phi^{P} n_{r} \mathrm{~d} l, \text { for } r \text { direction, } \\
\iint_{\Omega}\left(p \phi_{z}^{P}-\rho g \phi^{P}\right) \mathrm{d} s-\int_{\partial \Omega} p \phi^{P} n_{z} \mathrm{~d} l, \text { for } z \text { direction, }
\end{array}\right.
\end{gathered}
$$

where the superscripts $P$ and $Q$ are both node numbers. Then, the fluid velocity and nodal position are updated with an explicit integral scheme. The detailed mathematical formulation and numerical procedure are given by $\mathrm{Wu}$ and $\mathrm{Gu}$ [45].

During this phase, no mass or energy flux are transported between adjacent elements. Thus, the material density and the specific internal energy $e_{i n}$ are updated with

$$
\frac{\mathrm{d} \rho}{\mathrm{d} t}=-\rho\left(\nabla \cdot \mathbf{u}+\frac{1}{r} u\right)
$$

and

$$
\frac{\mathrm{d} \rho e_{i n}}{\mathrm{~d} t}=-\left(p+\rho e_{i n}\right)\left(\nabla \cdot \mathbf{u}+\frac{1}{r} u\right) .
$$

At this moment, the Lagrangian step is finished and the mesh is advanced forward with the material.

\subsection{Eulerian phase for multi-phase flow}

\subsubsection{Variables convection}

After the Lagrangian phase, the deformed mesh must be moved back to its original position to maintain the mesh quality. This is done by the Eulerian phase, in which the deformed mesh is moved back with the material fixed at the advanced position. During this process, the relative movement between the mesh and the fluid material is easy to obtain, and the convected volume is integrated over the shared edges between adjacent elements. The element-centered variables $\rho$ and $e_{i n}$ are then convected with the monotonic upwind scheme for conservation laws (MUSCL) $[41,46]$. The nodecentered momentum $\rho \mathbf{u}$ is convected with the half-index-shift scheme proposed by Benson [42]. 
Figure 3: Configuration for the construction of interface in a multi-phase element.

Because bubble motion in water is a typical multi-phase problem, the first task is to calculate the convected volume between adjacent elements while the deformed mesh is moving back to its original position, which is solved herein using the VOF method. As for the edge $e$ shared by two adjacent elements fully filled by the same fluid, the calculation of the transported volume is straight forward: $\delta=\int_{e} \mathbf{u} \cdot \mathbf{n d} l$. The difficult part is how to treat the multi-phase elements. In this paper, the second order interface construction method is employed in which the fluid interface is constructed with piecewise linear lines. The location and orientation of the interface is determined as below. Take a multi-phase element $i$ as an example as shown in Fig.3 in which the red curve is the actual interface and the dash segments are the piecewise linear approximations. The linear interface in element $i$ is then be written as

$$
n_{r} r+n_{z} z+b=0
$$

where the unit normal of the interface $\mathbf{n}=\left(n_{r}, n_{z}\right)$ points from fluid 2 to fluid 1 , and 
$b$ is an unknown constant. Firstly, the normal vector $\mathbf{n}$ is calculated with the fact

$$
\mathbf{n}=\frac{\nabla f_{1}\left(r_{c}, z_{c}\right)}{\left|\nabla f_{1}\left(r_{c}, z_{c}\right)\right|},
$$

where $f_{1}$ is the quadratic function fitting the element center values of all adjacent elements of element $i$, and $\left(r_{c}, z_{c}\right)$ is the location of the element center. In this paper, it is chosen as

$$
f_{1}(r, z)=\mathbf{K} \cdot \mathbf{X},
$$

where $\mathbf{K}=\left[k_{0}, k_{1}, k_{2}, k_{3}, k_{4}, k_{5}\right]^{T}$ is the coefficient vector and $\mathbf{X}=\left[r^{2}, z^{2}, r z, r, z, 1\right]^{T}$ is the coordinate vector. The coefficient vector $\mathbf{K}$ is calculated with the least square method by solving the following linear equations

$$
\left(\sum_{j=0}^{N_{e}} \mathbf{X}_{j} \otimes \mathbf{X}_{j}\right) \mathbf{K}=\sum_{j=0}^{N_{e}} \mathbf{X}_{j} f_{j} .
$$

Here $\mathbf{X}_{j}$ is the coordinate vector at the center of element $j, \otimes$ is the out product operator, and the summation is taken over all the elements adjacent to element $i$ including itself. Once $\mathbf{K}$ is obtained, the gradient of $f_{1}$ at $\left(r_{c}, z_{c}\right)$ and the normal vector of the interface can be easily calculated. As for the calculation of constant $b$ in Eq.(11), an iterative procedure is employed based on the fact

$$
\frac{\iint_{S_{1}} r d s}{\iint_{S} r d s}=f_{1},
$$

where $S$ is the area of the element, and $S_{1}$ is the area of the part occupied by fluid 1, as shown by the shadow region in Fig.3. Here, an iterative method is used to get the value of $b$.

The convected volume of each phase is calculated geometrically with the given nodal velocity and the intercept of the interface on the element edges. The underlying theoretical and numerical formulas are given in Hirt and Nichols [44], Youngs [47], and Tian et al. [37]. After the transported volume is calculated, the MUSCL algorithm[41, 46] and the Half Index Shift(HIS) scheme[41, 42] are used to transport 
the element-centered and nodal centered variables, respectively. Note that mass and internal energy are stored at the element centers while momentum is stored at nodes.

\subsubsection{Equations of states and material constants}

At the end of the Eulerian phases, the pressure $p$ needs to be updated with the fluid EoS for the next increment. Herein, the surrounding water is modeled with the Tammann equation[36] that reads

$$
p=\rho e_{i n}(\gamma-1)-\gamma P_{w}
$$

where $\gamma$ is the specific heat ratio and $P_{w}$ is the reference pressure. The gaseous explosion products inside the bubble is modeled with the JWL equation[48], namely

$$
p=A\left(1-\frac{\omega \rho}{R_{1} \rho_{0}}\right) e^{-\frac{\rho_{0}}{\rho} R_{1}}+B\left(1-\frac{\omega \rho}{R_{2} \rho_{0}}\right) e^{-\frac{\rho_{0}}{\rho} R_{2}}+\omega \rho e_{i n}
$$

in Sect.2.7 and Sect.3. Here, $\omega, A, B, R_{1}$, and $R_{2}$ are the material constants given in Table 2, and $\rho_{0}$ is the mass density of the explosive. The explosive is taken as Pentaerythritol Tetranitrate(PETN). If $\rho / \rho_{0}<<1$ and taking $\gamma=1+\omega$, Eq. (17) can be simplified as the EoS of an ideal gas, namely

$$
p=(\gamma-1) \rho e_{i n}
$$

\subsection{Non-dimensionalization}

The problem under investigation is non-dimensionalized to maintain uniform accuracy for simulating cases with different scales. The length scale is chosen as the expected maximum radius of the bubble expanding in the free field. Specifically, it can be calculated with the following semi-empirical formula[3] for UNDEX bubbles:

$$
R_{m}=3.38\left(\frac{W}{h+10.3}\right)^{\frac{1}{3}}
$$

where $W$ and $h$ are the equivalent TNT weight and initial depth of the explosive in units of kilograms and meters, respectively. The pressure and density scales are taken 
Table 1: Scales of different quantities for non-dimensionalization

\begin{tabular}{cccccc}
\hline \hline Quantity & Velocity & Acceleration & Time & Mass & Energy \\
\hline Scale & $\sqrt{P_{\infty} / \rho_{f}}$ & $P_{\infty} /\left(R_{m} \rho_{f}\right)$ & $R_{m} \sqrt{\rho_{f} / P_{\infty}}$ & $\rho_{f} R_{m}^{3}$ & $P_{\infty} R_{m}^{3}$ \\
\hline \hline
\end{tabular}

as $P_{\infty}$ and the surrounding fluid density $\rho_{f}$, respectively. Here, $P_{\infty}=P_{a t m}+\rho_{f} g h$ represents the undisturbed static ambient pressure at the same depth as the bubble center and $P_{a t m}$ is the atmospheric pressure. The scales of the other quantities can then be calculated from these three basic scales subsequently, as shown in Tab.1.

With these non-dimensional scales, the cases parameters can be non-dimensionalized, such as the strength parameter $\varepsilon=P_{0} / P_{\infty}$, the buoyancy parameter $\delta=\sqrt{g \rho R_{m} / P_{\infty}}$ and the initial non-dimensional radius $R_{0}^{*}=R_{0} / R_{m}$.

\subsection{Model validation by comparing with experiment}

The motion of an UNDEX bubble generated by a small explosive charge is simulated to validate the simulation of the bubble evolution compared with the experimental results given by Cui et al. [49] in which the evolution of the bubble is recorded by a high speed camera. The bubble is generated by a $4.0 \mathrm{~g}$ PETN charge detonated at a water depth of $1.0 \mathrm{~m}$. Its TNT equivalence is $5.2 \mathrm{~g}$ and the expected maximum radius $R_{m}$ calculated with Eq.(19) is $0.261 \mathrm{~m}$.

In the numerical simulation, the gaseous explosion products are modeled with the JWL EoS with the material constants given by Lorenz et al. [50] and listed in Tab.2 where $e_{i n 0}$ is the initial specific internal energy. Meanwhile, water is modeled with Tammann equation with $\gamma=7.15, P_{w}=330.9 \mathrm{MPa}$ and $\rho=1000 \mathrm{~kg} / \mathrm{m}^{3}$. The computation domain is taken as a rectangle of size $5 R_{m} \times 10 R_{m}$. The mesh size $L_{e}=0.01 R_{m}$. The simulation was performed on a personal desktop with the CPU of intel i7-8700 $(3.2 \mathrm{GHz} \times 6)$ and $16 \mathrm{~Gb}$ RAM. The code is paralleled with OpenMP API and took about 3 hours to complete a single simulation.

Table 2: Material properties of PETN[50].

\begin{tabular}{cccccccc}
\hline \hline & $\rho_{0}\left(\mathrm{~kg} / \mathrm{m}^{3}\right)$ & $\omega$ & $A(\mathrm{GPa})$ & $B(\mathrm{GPa})$ & $R_{1}$ & $R_{2}$ & $e_{i n 0}(\mathrm{MJ} / \mathrm{kg})$ \\
\hline PETN & 1762 & 0.57 & 1029.5 & 90.68 & 6.0 & 2.6 & 6.13 \\
\hline \hline
\end{tabular}


The results of the bubble evolution from the present model and the experiment are both shown in Fig.4 for comparison. In the first row, the bubble expands quickly after the detonation and reaches its maximum volume at $t=21.60 \mathrm{~ms}$ and then begins to collapse. Because of both the gravity and the high-pressure region below the bubble, the lower part of the bubble collapses faster than the other parts and develops a liquid jet that penetrates the bubble at $t=44.13 \mathrm{~ms}$ and reaches its minimum volume at the same time. The second row in Fig.4 shows the second cycle of the pulsation, in which the bubble presents more-complex phenomena. The toroidal bubble continues expanding while the jet keeps rushing into the fluid above the bubble and is slowed down by the upper high-pressure region. A necking effect emerges because of the push of the jet, which turns the bubble into an onion-like shape. When the bubble starts collapsing, its neck collapses first and splits the head of the neck apart from the main bubble so that the bubble loses some volume. This is another energy dissipation mechanism and was not clarified in previous studies by the author's knowledge. At the end of the second cycle, the lower part of the bubble is pressed by a ring-shape high-pressure region to form a onion-like shape as the beginning of this cycle but has the opposite direction.

Mostly, the simulated results match the experiment well. As for the vertical migration of the bubble center in the second pulsating cycle, the simulated results are less than the experimental ones, which is attributed to the neglect of the viscosity of the water. When the bubble is penetrated by the upward jet, the speed of jet is much higher than the surrounding fluid so that great velocity gradient appears. Physically, the viscosity of the fluid will lead to turbulence and slow down the jet so that the bubble is dragged upward to maintain the conservation of the vertical momentum of the system. Because this effect only plays an important role in a very local region where the jet rushes into the above water, the pulsating process is not affected as the migration by the ignorance of viscosity. Thus, the maximum volume of the bubble and the time when the second pulsating cycle ends still agree with the experimental results, which also indicates that the viscosity is trivial for the energy dissipation of the pulsating bubble.

The time histories of the equivalent radius $R=\left(\frac{3}{4} \frac{v_{b}}{\pi}\right)^{1 / 3}$ and mass of the internal 

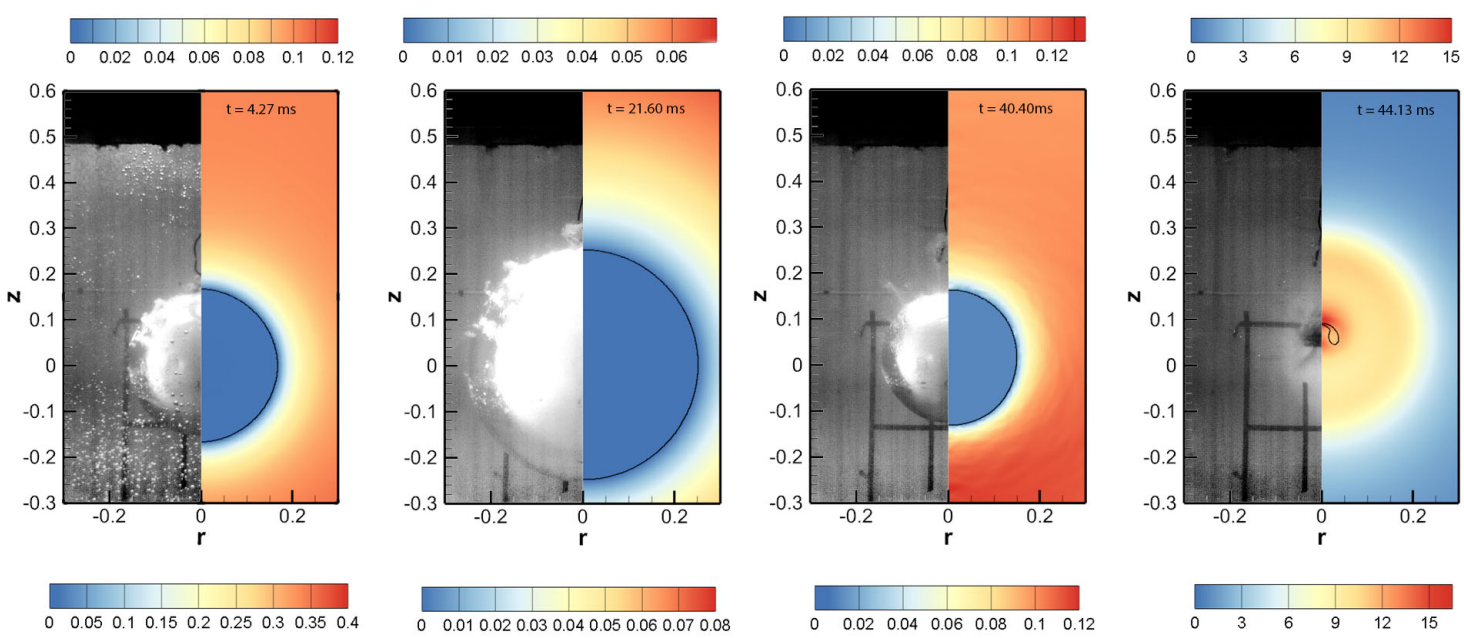

28
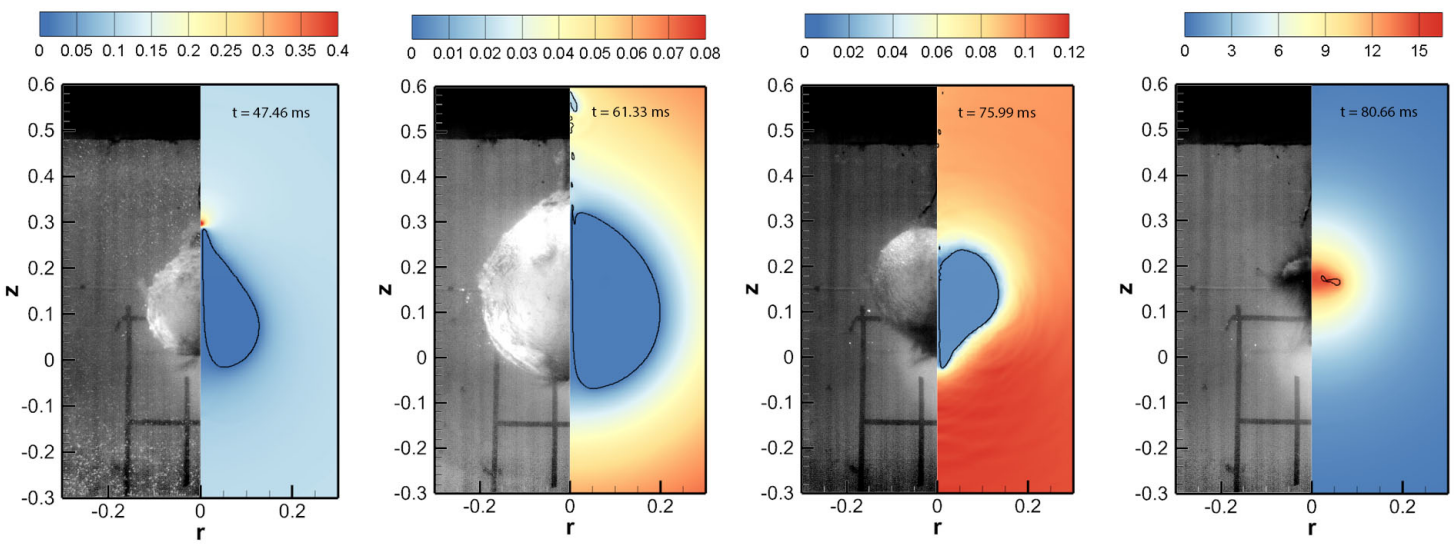

Figure 4: Evolution of an underwater explosion bubble for the first two oscillating cycles generated by a $4 \mathrm{~g}$ PETN explosive detonated at a depth of $1 \mathrm{~m}$. The comparison is made between the results from the present model(right) and the experiment(left)[49] at a sequence of time in ms. The color contour represents the pressure with the unit of MPa and the length unit is $\mathrm{m}$. 
gas products are shown in Fig. 5 where $v_{b}$ is the volume of the bubble. We can see that there are fluctuations of $2.5 \%$ of the total mass during the first 2 pulsating cycles from $4 \mathrm{~g}$ to $4.1 \mathrm{~g}$. The mass increases gradually during the expansion in each pulsating cycle and decrease in the collapsing phase. Then sudden jumps appear when the bubble reaches its minimum volume and penetrated by the jet. The most important reason for the non-conservation of the mass is that the volume fraction function $f$ mentioned in Sect.2.5 must be cutoff to be within the range of $[0,1]$ based on its the physical meaning after each Eulerian phase which can be decreased by the refinement the mesh. Thus, the present model conserves in mass and has proper accuracy to investigate the energy dissipation of the pulsating bubble.

Except for the EFEM presented in this work, the Geers-Hunter model[24, 32] and Gilmore equation[31] are also used to simulate the pulsating bubble for comparison. The Gilmore equation reads

$$
R \ddot{R}\left(1-\frac{\dot{R}}{c}\right)+\frac{3}{2} \dot{R}^{2}\left(1-\frac{\dot{R}}{3 c}\right)=\left(1+\frac{\dot{R}}{c}\right) H+\left(1-\frac{\dot{R}}{c}\right) \dot{H}
$$

where

$$
H=\frac{c^{2}-c_{\infty}^{2}}{\gamma-1}
$$

is the enthalpy at the bubble wall; The local sound speed $c[51]$ is calculated with the following equation

$$
c^{2}=\frac{\gamma}{\rho}\left(p_{b}+P_{w}\right)
$$

and $c_{\infty}$ is the sound speed of water at pressure of $P_{\infty}$, where $p_{b}$ is the average pressure $p$ at the bubble wall. Ignoring the contribution of viscosity and surface tension, according to Dobratz [52] $p_{b}$ is approximated by the adiabatic JWL EoS

$$
p_{b}=A \mathrm{e}^{-\left(\frac{R}{R_{0}}\right)^{3} R_{1}}+B \mathrm{e}^{-\left(\frac{R}{R_{0}}\right)^{3} R_{2}}+C\left(\frac{R}{R_{0}}\right)^{3(1+\omega)},
$$

and

$$
C=\omega \rho_{0} e_{i n 0}-A \frac{\omega}{R_{1}} \mathrm{e}^{-R_{1}}-B \frac{\omega}{R_{2}} \mathrm{e}^{-R_{2}}
$$


One can refer to [24] and [32] for the detailed formulation of the Geers-Hunter model. Both of the two spherical bubble model are solved with the explicit 4th order RungeKutta method with the same initial conditions and material constants as those given in the simulation using the present EFEM. The radius histories are also plotted in Fig. 5. Although the Gilmore's solution is very accurate for collapsing cavity bubble[53], the maximum radii of the first two pulsating cycles calculated with the Gilmore equation is much smaller than the experimental ones for this UNDEX bubble case.

The Gilmore equation is based on the Kirkwood-Bethe assumption which assumes that the quantity $r\left(H+\frac{1}{2}|\mathrm{u}|^{2}\right)$ is a constant along with an outward going characteristic in the surrounding water.[53] When it is used to simulate a collapsing bubble with initial pressure under $P_{\infty}$, the assumption is reasonable because the initial velocity jump is much smaller than the sound speed $c_{\infty}$ and is ignorable. However, in a UNDEX bubble case, the jump is comparable to $c_{\infty}$ because of an extremely high initial pressure and the shock wave amplitude is overestimate when the Kirkwood-Bethe assumption is used[3]. As a result, the energy that is to support the bubble expansion is dissipated through the shock wave and a smaller maximum radius is obtained. As for the Geers-Hunter model, the result of bubble radius history matches that of the present model well. But we should note that the shock wave phase is obtained by using an empirical formula other than solving a differential equation, which avoids the difficulty about the initial velocity jump. In the three radius histories, the maximum radii in the second cycles are about $80 \%$ of that in the first ones. Thus, when the dissipated energy during the first collapse is concerned, all the three approaches can derive acceptable results.

\section{Energy dissipation mechanism of UNDEX bubbles}

The total energy of the system of bubbles can be expressed as[3]

$$
E=E_{k}+\frac{4}{3} \pi R^{3}\left(P_{\infty}-\rho g z_{m}+\rho \bar{e}_{i n}\right),
$$

where $E_{k}$ is the kinetic energy of the fluid field, $R$ is the equivalent radius of the bubble, $z_{m}$ is the migration distance of the bubble center in the vertical direction, 
Figure 5: Time histories of the equivalent radius and mass of the internal gas for the UNDEX case in Fig.4.

and $\bar{e}_{i n}$ is the average specific energy of the internal gas. The first term on the right-hand side is the kinetic energy of the fluid. The first two terms in the brackets represent the gravity potential energy of the surrounding fluid, and the third term is the internal energy of the gas inside the bubble. Note that the internal energy of the surround water is neglected because it's actually not part of the energy of the system. Usually, the pressure wave around the bubble propagates outwards, and the energy associated with the wave should be considered as dissipated. When the bubble reaches its maximum radius, the velocity of the surrounding flow is small enough to be ignored so that the kinetic energy in Eq. 25 is about zero. Besides, the change in $e_{i n}$ is also small if the maximum radius exceeds the balanced radius. Therefore, we can relate the remaining energy of the system approximately to the maximum radius cubed. In another words, a reduced maximum radius represents energy dissipation in the system. As shown in Fig.5, the maximum radius in the second cycle is $0.2 \mathrm{~m}, 80 \%$ of the first one, which indicates that the dissipated energy around the first minimum volume is about $50 \%$ of the total energy at the first maximum radius. By plotting the histories of different kinds of energy in Fig.6, the statement above can be easily verified.

Most researchers agree that the compressibility of the surrounding fluid is one 
of the dominant factors in the complex energy dissipation mechanisms. When the bubble pulsates in a compressible fluid, pressure waves carrying energy radiate away from the bubble. This part of the energy is subsequently lost into the surrounding fluid and seldom returns to the bubble. Besides, the nonspherical collapsing bubble also emits shock waves and loses energy when the jet impact occurs[54]. Therefore, the energy dissipation due to the compressibility of the surrounding fluid is also regarded as a wave effect.

Another important reason for energy dissipation is the nonspherical motion of the bubble. Under the effects of gravity or nearby boundaries, the bubble moves nonspherically, which leads to the surrounding fluid not be stationary when the bubble reaches its maximum and minimum radii, and possesses considerable kinetic energy. This energy part is extracted from the potential energy that is supposed to support the expansion of the bubble, thereby resulting in reduced maximum radius. This part of the dissipated energy can be calculated approximately as follows. In the far field of the UNDEX, the disturbance of the fluid particle is so small that the problem can be linearized with the acoustic assumption. Thus, the part of the energy lost due to wave effects can be approximated by the acoustic wave energy that propagates away, namely

$$
E_{w}(t)=\frac{4 \pi}{\rho_{\infty} c_{\infty}} \int_{0}^{t} R_{j}^{2} \delta p_{j}^{2}\left(t+t_{d}\right) \mathrm{d} t,
$$

where $\rho_{\infty}$ is the density of the fluid under pressure $P_{\infty}, R_{j}$ is the distance of the field point from the bubble center, $t_{d}=R_{j} / c_{\infty}$ is the time delay, and $\delta p_{j}$ is the linearized pressure over the ambient pressure $P_{\infty}$ and is calculated from[32]

$$
\delta p_{j}\left(t+t_{d}\right)=\frac{\rho_{\infty}}{R_{j}}\left(\frac{R_{0}}{R_{j}}\right)^{\alpha}\left[R(\tau)^{2} \ddot{R}(\tau)+2 R(\tau) \dot{R}^{2}(\tau)\right],
$$

where $\tau=\left(R_{0} / R_{j}\right)^{\beta}$ t. $\alpha$ and $\beta$ are the material constants of the explosive and change the pressure history linearly in terms of amplitude and period. Substituting Eq. (27) into Eq. (26) gives

$$
\dot{E}_{w}=K \frac{\rho_{\infty}}{c_{\infty}}\left(R^{2} \ddot{R}+2 R \dot{R}^{2}\right)^{2}
$$


where the factors with exponents $\alpha$ and $\beta$ are both dropped and their effects are represented in the constant $K$ after the integration. The argument $t$ is omitted in $E_{w}$ and $R$. Thus, the wave effects are related linearly to the wave speed $c_{\infty}$.

Thermal conduction and fluid viscosity also contribute to the energy dissipation. In the most bubble-dynamics research focusing on the first pulsation cycle, they are usually ignored because their influences are small. When the bubble collapses to its minimum radius, the Rayleigh-Taylor instability caused by the misalignment between the pressure and density gradients leads to micro-jets penetrating the bubble.[55] This can amplify significantly the effects of thermal conduction and viscosity on the energy dissipation. However, because of the assumption of axisymmetry, it is not included in the present model. We should note that even with a fully 3-dimensional model, the simulation is still nontrivial because the micro-jets are on a scale far smaller than the bubble and an extremely fine mesh would be required.

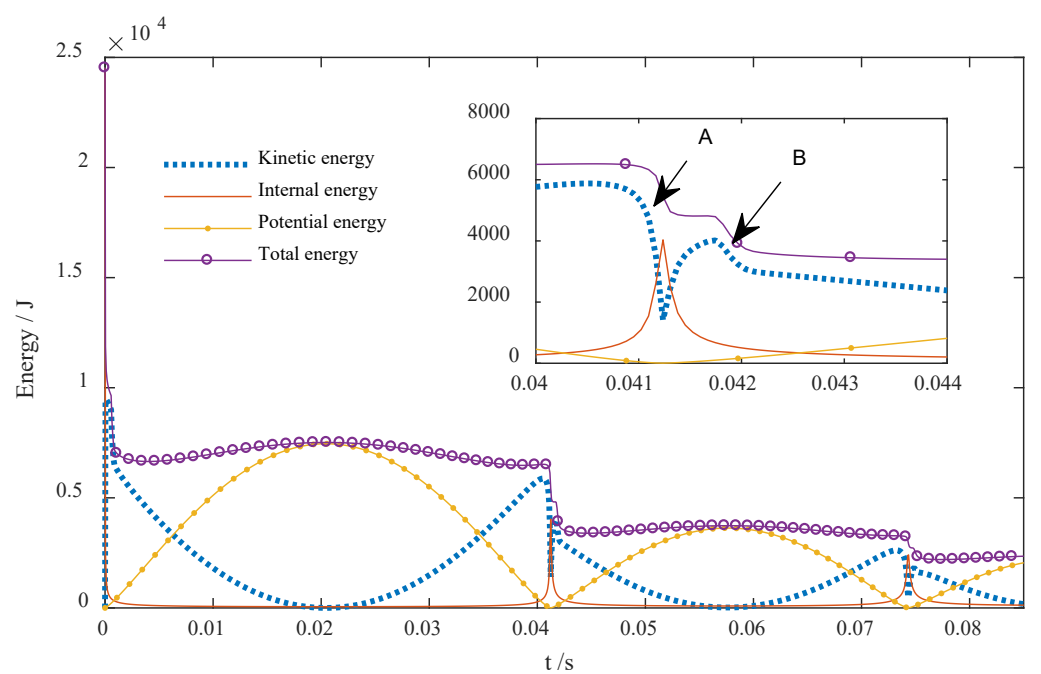

Figure 6: Time histories of the energy components of the bubble system for the case in Fig.4.

Fig. 6 shows the histories of the energy components of the bubble system calculated using the present model, namely the kinetic energy, the internal energy of the gas, the potential energy of the surrounding water, and the total energy. The total energy experiences two steep decreases around its minimum volume in each pulsating 
cycle, as denoted by A and B in Fig. 6. The decrease A is due to energy conversion from internal energy to pressure wave energy propagating away in the surrounding water. It is noted that the internal energy of the pressure wave in the surrounding fluid is not considered in the total energy. The decrease B is attributed to the escape of the pressure wave from the computational domain. Quantitatively, the first expansion of the bubble emits a shock wave into the water, resulting in an energy dissipation of roughly $70 \%$ of the initial total energy. The percentage of energy lost in each subsequent bubble pulsation cycle decreases significantly, being roughly $50 \%$ and $38 \%$ for the first and second rebounds of the bubble, respectively. For the kinetic energy, the history curve fluctuates rapidly around the moments when the bubble reaches its minimum volume. In the bubble dynamics models based on a spherical assumption, such as the Geers-Hunter and Rayleigh-Plesset models, the kinetic energy is expressed as $2 \pi R^{3} \dot{R}^{3}$. When the bubble reaches its minimum volume, the rate of change $\dot{R}$ of the equivalent radius is zero. Thus, the kinetic energy is also zero. However, if the nonspherical motion of the bubble is considered, the kinetic energy does not decrease to zero because the surrounding fluid is not stationary when the bubble reaches its minimum volume. As shown in Fig. 6, the kinetic energy is $1.41 \mathrm{~kJ}$, about $25 \%$ of the total energy at $t^{*}=0.0413$.

\section{Bubble dynamics in compressible fluids}

It is shown that the wave-energy dispersion rate is related linearly to $c_{\infty}^{-1}$ and increases rapidly with the growing velocity and acceleration by Eq. (28). Because most of the energy dissipation occurs near the bubble's minimum radius, the initial pressure difference across the bubble surface is crucial to the energy dissipation. To examine how compressibility influences the bubble dynamics, a new form of the Mach number is used to include these two factors, namely

$$
M_{a}=\frac{1}{c} \sqrt{\frac{P_{0}-P_{\infty}}{\rho_{\infty}}} .
$$


Physically, $M_{a}$ represents the maximum Mach number of the problem. Given that most energy dissipation due to wave effects occurs during the very short period at the beginning of the bubble pulsation, when the initial pressure dominates the problem, it is reasonable to use $M_{a}$ to evaluate the energy-dissipation effect in the analysis. For the UNDEX bubble generated by a specific type of explosive, the initial pressure $P_{0}$ is constant and usually much higher than $P_{\infty}$. The density $\rho$ and the sound speed $c$ vary in relatively small ranges for water under different natural circumstances.

The change in $M_{a}$ is thus related to explosive types or surrounding fluid. In the present study, how $M_{a}$ influences energy dissipation is investigated by two means. One is to change the initial pressure $P_{0}$, which represents a change in the explosive. The other is to change the sound speed $c_{\infty}$, which represents the bubble pulsating in different fluids.

\subsection{Influence of compressibility with different initial pressure}

In this section, six cases with different values of the strength parameter $\varepsilon$ are simulated to demonstrate the influence of initial pressure. The case parameters are modified from those of $500 \mathrm{~kg}$ of TNT detonated at a depth of $100 \mathrm{~m}$. The expected maximum radius $R_{m}$ is $5.6 \mathrm{~m}$, and the internal gas is modeled with the ideal gas EoS with the initial conditions given by Tab.3. The bubble evolution of case 4 is shown in Fig. 7 in which all variables are non-dimensionalized with the scales listed in Tab.1.

The first pulsation cycle is similar to the case shown in Fig. 4, and Fig. 7 starts from the moment of penetration. After penetration, the necking effect and the highpressure region emerge above the bubble. Because the buoyancy parameter exceeds that in the previous case, the jet is wider and the necking effect is stronger. During the collapsing phase of this cycle, the ring-shaped high-pressure region below the bubble also presses on the lower part of the bubble and splits it eventually. This fragmentation generates an impact pressure wave, and the main part of the bubble continues to collapse.

Six cases with the same initial conditions are studied with $\varepsilon=10,20,50,100$, 200, 400 and $c_{\infty}=1540 \mathrm{~m} / \mathrm{s}$ for these cases specifically. The other corresponding 

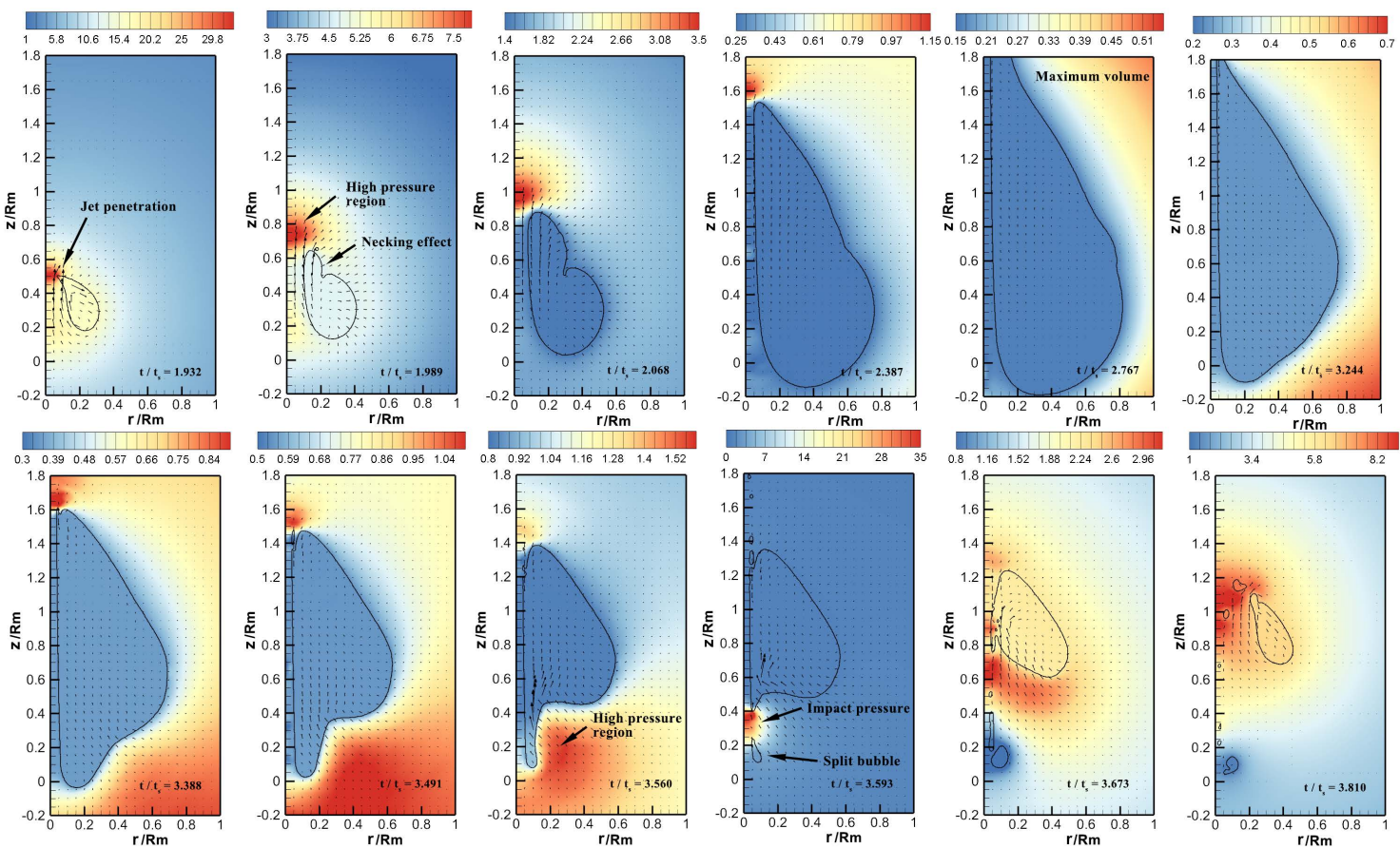

Figure 7: Bubble evolution generated by $500 \mathrm{~kg}$ of TNT detonated at a depth of $100 \mathrm{~m}$ with $\varepsilon=100$ and $R_{0}^{*}=0.2043$. The color contours represent the non-dimensional fluid pressure and the arrows indicate the fluid velocity. Only the right half of figures are rendered because of axisymmetry. $t_{s}$ represents the time scale in Tab.1.

non-dimensional parameters are listed in Table 3.

Table 3: Non-dimensional case parameters with differing $\varepsilon$.

\begin{tabular}{cccc}
\hline \hline Case & $\varepsilon$ & $R_{0}^{*}$ & $M_{a}$ \\
\hline 1 & 10 & 0.3533 & 0.0616 \\
2 & 20 & 0.2695 & 0.0895 \\
3 & 50 & 0.1911 & 0.1437 \\
4 & 100 & 0.1485 & 0.2043 \\
5 & 200 & 0.1159 & 0.2897 \\
6 & 400 & 0.0908 & 0.4102 \\
\hline \hline
\end{tabular}

Fig. 8 shows the histories of the nondimensional bubble pulsation radii with differing initial strength parameter $\varepsilon$. Because Eq. (2) is based on the incompressible assumption and ignores wave effects, the bubble whose initial condition is calculated 
Figure 8: Time histories of bubble radius with differing strength parameter $\varepsilon$ for the first two pulsation cycles.

with Eq. (2) cannot reach the expected maximum radius. A higher strength parameter represents a higher initial pressure inside the bubble, which will drive the bubble to expand more quickly and radiate more energy. As a result, the bubble will expand to a smaller maximum radius. Thus, with increasing $\varepsilon$, the pulsation period and the decrease in maximum radius increase accordingly as shown in Fig. 8, which indicates that more energy is dissipated through the emitted pressure wave.

The diamond markers in Fig. 8 represent the moments when the bubbles are penetrated by the liquid jets. Penetration is clearly delayed with decreasing $\varepsilon$; for $\varepsilon<50$, it occurs in the expansion phase of the second pulsation cycle. It is noted that the jet development of the UNDEX bubble is dominated by the gravity as represented by the buoyancy parameter $\delta=\sqrt{g \rho R_{m} / P_{\infty}}$. However, because these cases have the same $\delta$, the difference in penetration time must be attributed to other factors. Table 3 shows that the initial radius is greater for a smaller strength parameter. Thus, the corresponding minimum radius at the end of the first pulsation cycle is greater. This results that the bubble starts to rebound, and that the top of 
the bubble tends to move upward before the upward jet can reach it. Consequently, the penetration occurs in the second pulsation cycle for small $\varepsilon$. The bubble profiles at penetration are plotted in Fig. 9 for comparison.

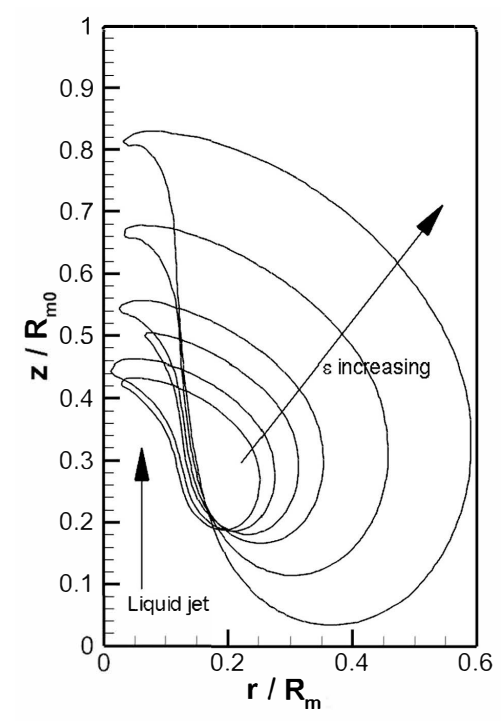

Figure 9: Bubble profiles when penetrated by jets with $\varepsilon=10,20,50,100,200$, and 400 .

\subsection{Influence of compressibility with differing sound speed}

To investigate how the speed of sound influences the fluid, six cases with differing $P_{w}$ are chosen. Furthermore, to be comparable with the influence of initial pressure, the Mach number $M_{a}$ is chosen to be the same as that in Section 4.1. The strength parameter $\varepsilon$ is taken as 100 for all six cases. Then, $P_{w}$ and $c_{\infty}$ are calculated using Eqs. (29) and (22) as given in Table 4. The bubble radius histories are compared in Fig. 10, which shows a similar reduction in maximum radius with $M_{a}$, corresponding to different values of the strength parameter $\varepsilon$. Although these cases have the same $\varepsilon$ and initial radius, the minimum radius that the bubble can achieve at the end of the first pulsation cycle differs significantly because of the differing extent of the energy dissipation. Thus, penetration occurs in the first cycle in all six cases, which differs from the cases with varying $\varepsilon$. 


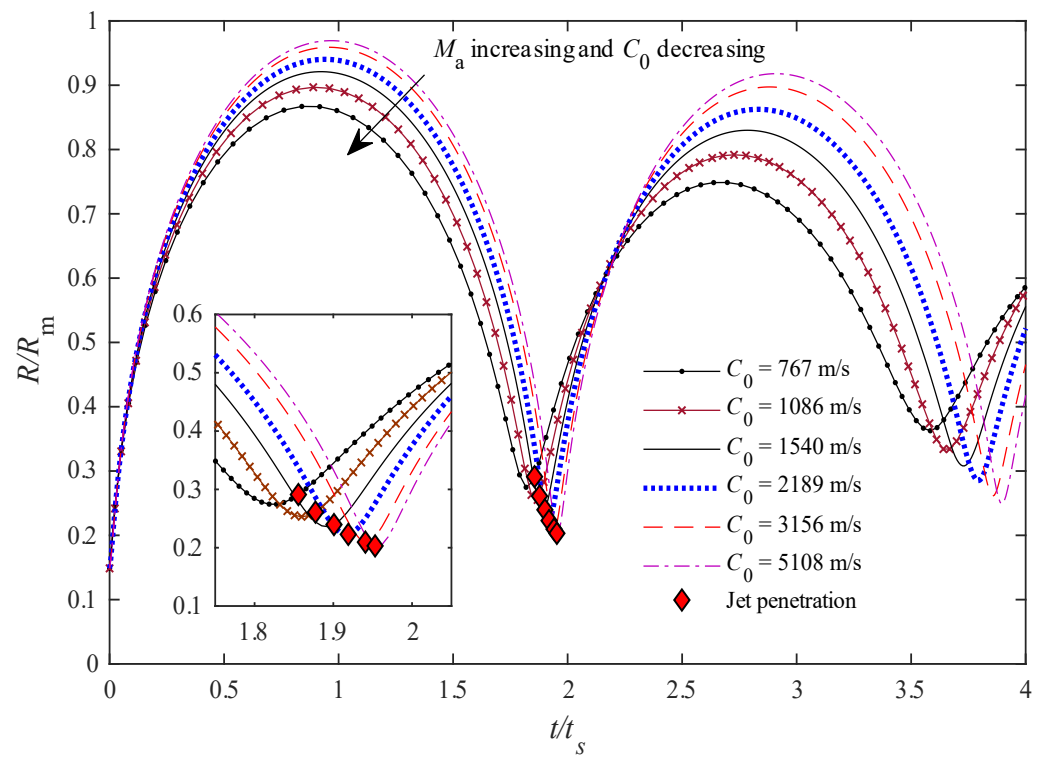

Figure 10: Histories of bubble radius with different sound speeds for the first two pulsation cycles.

Table 4: Nondimensional parameters with differing $c_{\infty}$.

\begin{tabular}{cccc}
\hline \hline Case & $c_{\infty}(\mathrm{m} / \mathrm{s})$ & $P_{w}(\mathrm{MPa})$ & $M_{a}$ \\
\hline 1 & 5,108 & $3,647.6$ & 0.0616 \\
2 & 3,516 & $1,727.6$ & 0.0895 \\
3 & 2,189 & 669.2 & 0.1437 \\
4 & 1,540 & 330.9 & 0.2043 \\
5 & 1,086 & 164.0 & 0.2897 \\
6 & 767 & 81.3 & 0.4102 \\
\hline \hline
\end{tabular}

When the bubble expands to its maximum radius, the kinetic energy can be ignored because the velocity of the surrounding fluid is very small. Assuming that the bubble center migrates by a distance that is negligible compared to the initial depth $h$ in free-field UNDEX problems, the internal energy of the internal gas products can be approximated by the work applying on the surrounding water during the bubble 
expanding from $r=R_{m}$ to $r=\infty$, namely

$$
E_{i n}=4 \pi \int_{R}^{\infty} r^{2} C\left(\frac{R_{0}}{r}\right)^{3+3 \omega} \mathrm{d} r=\frac{4 \pi}{3 \omega R_{m}^{3 \omega}} C R_{0}^{3+3 \omega}
$$

where the exponential terms in the adiabatic JWL EoS Eq. (23) are neglected because $r \geq R_{m}$ and $R_{m}$ is far greater than $R_{0}$ such that the exponential index $-\left(\frac{R_{0}}{r}\right)^{3+3 \omega} R_{1}$ and $-\left(\frac{R_{0}}{r}\right)^{3+3 \omega} R_{2}$ are significantly smaller than 0 . For instance, in the UNDEX case shown in Sect.2.6, the maximum radius $R_{m}$ is about 30 times of the initial radius $R_{0}$. Thus, Eq. (25) can be simplified to approximate the remaining energy $E_{r}$ of the system as

$$
E_{r}=\frac{4}{3} \pi R_{m}^{3}\left[P_{\infty}+\frac{C}{\omega}\left(\frac{R_{0}}{R_{m}}\right)^{3(1+\omega)}\right]
$$

for the JWL EoS or

$$
E_{r}=\frac{4}{3} \pi R_{m}^{3} P_{\infty}\left[1+\frac{\varepsilon}{\omega}\left(\frac{R_{0}}{R_{m}}\right)^{3 \gamma}\right]
$$

for the ideal gas EoS and $R_{c}$ is the radius of the spherical explosive. Eq. (31) gives an approach for obtaining the energy lost in one pulsation cycle. From Eq. (31), how the percentage of the initial energy that remains changes with $M_{a}$ is shown in Fig. 11 and is compared for differing strength parameter. In Fig. 11, the curves for different $\varepsilon$ and $c_{\infty}$ clearly agree well with each other for both the maximum radius and the lost energy, thereby indicating that the nondimensional parameter $M_{a}$ presented herein can represent the wave effects of a pulsating bubble. Most of the differences come from the nonlinearity of the fluid EoS and increase with $M_{a}$. Within the range of $M_{a}$ analyzed, the maximum radius and lost energy are both related approximately linearly to $M_{a}$ with slopes of -0.30 and 0.65 , respectively. For the case presented in Section 3, $M_{a}$ is approximately 0.88 and the percentage of energy lost is roughly $60 \%$, which indicates a slope of 0.68 and also agrees with the linear relationship. Thus, this is a clear demonstration that the modified Mach number $M_{a}$ can be used to predict the energy dissipation caused by the wave effects of a pulsating bubble. For the second pulsation cycle, the prediction is less accurate because other energy 
Figure 11: Changes of nondimensional maximum radius and energy lost in the first pulsation cycle for differing $\varepsilon$ and $c_{\infty}$.

dissipation mechanisms, such as viscosity and thermal conduction accelerated by surface instability, participate in the pulsation process and play important roles in energy dissipation.

\section{Conclusion}

In this paper, UNDEX bubble dynamics in a compressible fluid are investigated numerically using EFEM. The VOF method and MUSCL are combined to solve the multi-phase problem. As for the validation, a small charge UNDEX case is simulated with the present model, and compared with the experimental results. The comparison shows the model has excellent accuracy in the first two bubble pulsation cycles which draw more attention than the subsequent cycles. The results of the same case from the Gilmore equation and Geers-Hunter model are discussed. It is found that they either neglect the discontinuous shock wave or approximate it by an empirical formula. As a result, artificial initial conditions are needed to produce reasonable results. Differing from the former two, the model built in this paper can 
resolve the shock wave propagation physically and correctly such that the physically meaningful initial condition can be determined directly. Thus, it can be used for analyzing the energy dissipation.

The simulation results of several different cases using the present model show that the nonspherical motion introduces two energy dissipation mechanisms. Firstly, it can lead to bubble fragmentation, and the energy of small fragments is considered lost because only the main bubble is usually concerned. Secondly, the jet impact during the collapse of the bubble emits a shock wave propagating away with energy. Besides, the nonspherical motion can also enhance the energy dissipation through viscosity because of the shear flow, which is not discussed in this paper.

The wave effect consumes more energy than the nonspherical motion. Within one pulsation cycle, the dissipated energy through the wave effect is linearly related to a modified Mach number $M_{a}$ which is proportional to the initial internal pressure of the bubble and the inverse of the sound speed of the surrounding fluid. Thus, it can be used to predict the energy dissipation of a pulsating bubble in a compressible fluid without performing a complete simulation.

\section{Acknowledgments}

The authors would like to acknowledge the support of the National Natural Science Foundation of China (Grant No. 51879050 and 51609044), the China Postdoctoral Science Foundation (Grant No. 2016M600244 and 2018T110271), the State Scholarship Fund of China(Grant No. 201906680004) and the Heilongjiang Postdoctoral Fund (Grant No. LBH-Z16043).

\section{References}

[1] L. Rayleigh. On the pressure developed in a liquid during the collapse of a spherical cavity. Philosophical Magazine, 34:94-98, 1917.

[2] P. Cui, A. M. Zhang, S. P. Wang, and B. C. Khoo. Ice breaking by a collapsing bubble. Journal of Fluid Mechanics, 841:287-309, 2018. 
[3] R. H. Cole. Underwater explosion. Princeton University Press, Princeton USA, 1948.

[4] E. Klaseboer, K. C. Hung, C. W. Wang, and B. C. Khoo. Experimental and numerical investigation of the dynamics of an underwater explosion bubble near a resilient/rigid structure. Journal of Fluid Mechanics, 53(7):387-413, 2005.

[5] N. Zhang, Z. Zong, and W. P. Zhang. Dynamic response of a surface ship structure subjected to an underwater explosion bubble. Marine Structures, 35: 26-44, 2014.

[6] A. M. Zhang and Y. L. Liu. Improved three-dimensional bubble dynamics model based on boundary element method. Journal of Computational Physics, 294: 208-223, 2015.

[7] J. Zheng, D. Zhang, B. H. Jiang, and F. Y. Lu. Formation mechanism of water jets induced by the interaction between bubble and free surface. Acta Physica Sinica, 66, 2017.

[8] K. L. de Graaf, I. Penesis, and P. A. Brandner. Modelling of seismic airgun bubble dynamics and pressure field using the Gilmore equation with additional damping factors. Ocean Engineering, 76:32-39, 2014.

[9] X. Huang, A. M. Zhang, and Y. L. Liu. Investigation on the dynamics of airgun array bubbles based on the dual fast multipole boundary element method. Ocean Engineering, 124:157-167, 2016.

[10] S. Zhang, S. P. Wang, A. M. Zhang, and Y. Q. Li. Numerical study on attenuation of bubble pulse through tuning the air-gun array with the particle swarm optimization method. Applied Ocean Research, 66:13-22, 2017.

[11] A. Dadvand, B. C. Khoo, M. T. Shervani-Tabar, and S. Khalilpourazary. Boundary element analysis of the droplet dynamics induced by spark-generated bubble. Engineering Analysis with Boundary Elements, 36(11):1595-1603, 2012. 
[12] A. Dadvand, M. Dawoodian, B. C. Khoo, and R. Esmaily. Spark-generated bubble collapse near or inside a circular aperture and the ensuing vortex ring and droplet formation. Acta Mechanica Sinica, 2013.

[13] J. R. Blake and D. C. Gibson. Cavitation bubbles near boundaries. Annual Review of Fluid Mechanics, 19:99-123, 1987.

[14] A. M. Zhang, S. Li, and J. Cui. Study on the splitting of a toroidal bubble near a rigid boundary. Physics of Fluids, 27:062102, 2015.

[15] Y. Tagawa and I. R. Peters. Bubble collapse and jet formation in corner geometries. Journal of Fluid Mechanics, 3:081601R, 2018.

[16] A. M. Zhang, P. Cui, J. Cui, and Q. X. Wang. Experimental study on bubble dynamics subject to buoyancy. Journal of Fluid Mechanics, 776:137-160, 2015.

[17] S. P. Wang, A. M. Zhang, Y. L. Liu, S. Zhang, and P. Cui. Bubble dynamics and its applications. Journal of Hydrodynamics, 30:975-991, 2018.

[18] C. Wang, B. C. Khoo, and K. S. Yeo. Elastic mesh technique for 3d bim simulation with an application to underwater explosion bubbles. Computers and Fluids, 32(9):1195-1212, 2003.

[19] Q. X. Wang, K. S. Yeo, B. C. Khoo, and K. Y. Lam. Vortex ring modelling of toroidal bubbles. Theoretical Computational Fluid Dynamics, 19:303-317, 2005.

[20] Y. L. Liu, S. P. Wang, and A. M. Zhang. Interaction between bubble and air-backed plate with circular hole. Physics of Fluids, 28(6):1195-1212, 2016.

[21] N. Saleki-Haselghoubi and A. Dadvand. Simulation of bubble dynamics oscillating near a circular aperture made in a curved rigid plate using boundary element method. Ocean Engineering, 151:12-22, 2018.

[22] C. Wang and B. C. Khoo. An indirect boundary element method for threedimensional explosion bubbles. Journal of Computational Physics, 194:451-480, 2004. 
[23] L. T. Liu, X. L. Yao, A. M. Zhang, and Y. Y. Chen. Numerical analysis of the jet stage of bubble near a solid wall using a front tracking method. Physics of Fluids, 29:012105, 2017.

[24] T. L. Geers and K. S. Hunter. An integrated wave-effects model for an underwater explosion bubble. Journal of the Acoustical Society of America, 111(4): 1584-1601, 2002.

[25] A. M. Zhang, S. P. Wang, and G. X. Wu. Simulation of bubble motion in a compressible liquid based on the three dimensional wave equation. Engineering Analysis with Boundary Elements, 37:1179-1188, 2013.

[26] W. Xiao, A. M. Zhang, and S. P. Wang. Investigation of bubble dynamics of underwater explosion based on improved compressible numerical model. Applied Ocean Research, 59:472-482, 2016. ISSN 0141-1187.

[27] Q. X. Wang. Multi-oscillations of a bubble in a compressible liquid near a rigid boundary. Journal of Fluid Mechanics, 745(4):509-536, 2014.

[28] Q. X. Wang. Non-spherical bubble dynamics of underwater explosions in a compressible fluid. Phys. Fluids, 25(7), 2013.

[29] J. B. Keller and M. Miksis. Bubble oscillations of large amplitude. Journal of the Acoustical Society of America, 68(2):628-633, 1980.

[30] A. Lezzi and A. Prosperetti. Bubble dynamics in a compressible liquid. part 2. second-order theory. Journal of Fluid Mechanics, 185:289-321, 1987.

[31] A. Prosperetti and A. Lezzi. Bubble dynamics in a compressible liquid. part 1. first-order theory. Journal of Fluid Mechanics, 168:457-478, 1986.

[32] T. L Geers and C. K Park. Optimization of the GH bubble model. Shock and Vibration, 12(1):3-8, 2005.

[33] Q. X. Wang. Local energy of a bubble system and its loss due to acoustic radiation. Journal of Fluid Mechanics, 797:201-230, 2016. 
[34] E. Johnsen and T. Colonius. Numerical simulations of non-spherical bubble collapse. Journal of Fluid Mechanics, 629:231-262, 2009.

[35] N. A. Hawker and Y. Ventikos. Interaction of a strong shockwave with a gas bubble in a liquid medium: a numerical study. Journal of Fluid Mechanics, 701: 59-97, 2012.

[36] Y.L. Liu, A. M. Zhang, Z. L. Tian, and S. P. Wang. Investigation of free-field underwater explosion with eulerian finite element method. Ocean Engineering, 166:182-190, 2018.

[37] Z. L. Tian, Y. L. Liu, A. M. Zhang, and S. P. Wang. Analysis of breaking and re-closure of a bubble near a free surface based on the eulerian finite element method. Computers and Fluids, 170:41-52, 2018.

[38] C. F. Rowlatt and S. J. Lind. Bubble collapse near a fluid-fluid interface using the spectral element marker particle method with applications in bioengineering. International Journal of Multiphase Flow, 90:118-143, 2017.

[39] A. R. Jamaluddin, G. J. Ball, C. K. Turangan, and T. G. Leighton. The collapse of single bubbles and approximation of the far-field acoustic emissions for cavitation induced by shock wave lithotripsy. Journal of Fluid Mechanics, 677: 305-341, 2011.

[40] W. F. Noh. Cel: A time dependent two space-dimensional, coupled eulerian lagrangian code. In Methods of Computational Physics, 1964.

[41] D. J. Benson. Computational methods in Lagrangian and Eulerian hydrocodes. Computer Methods In Applied Mechanics And Engineering, 99:235-394, 1992.

[42] D. J. Benson. Momentum advection on unstructured staggered quadrilateral meshes. International Journal for Numerical Methods in Engineering, 75:15491580, 2008.

[43] S. P. Wang, W. Y. Duan, and Q. X. Wang. The bursting of a toroidal bubble at a free surface. Ocean Engineering, 109:611-622, 2015. 
[44] C. W. Hirt and B. D. Nichols. Volume of fluid method for the dynamics of free boundaries. Journal of Computational Physics, 39:201-225, 1981.

[45] S. R. Wu and L. Gu. Introduction to the explicit finite element method for nonlinear transient dynamics. John Wiley, Hoboken, New Jersey, 2012.

[46] B. Van Leer. Towards the ultimate conservative difference scheme iv: a new approach to numerical convection. Journal Of Computational Physics, 23:276299, 1977.

[47] D. L. Youngs. Numerical methods in fluid dynamics. Academic, New York, 1982.

[48] E. L. Lee, H. C. Hornig, and J. W. Kury. Adiabatic expansion of high explosive detonation products. Report UCRL-50422, University of California, 1968.

[49] P. Cui, A. M. Zhang, and S. P. Wang. Small-charge underwater explosion bubble experiments under various boundary conditions. Physics of Fluids, 28:117103, 2016.

[50] K. T. Lorenz, E. L. Lee, and R. Chambers. A simple and rapid evaluation of explosive performance - the disc acceleration experiment, 2014.

[51] E. F. Toro. Riemann solvers and numerical methods for fluid dynamics a pratical introduction. Springer, Berlin, 2009.

[52] B. M. Dobratz. LLNL explosives handbook: properties of chemeical explosives and explosive simulants. Technical Report UCRL-52997, Lawrence Livermore National Lab., 1981.

[53] R. Hickling and M. S. Plesset. Collapse and rebound of a spherical bubble in water. Physics of Fluids, 7:7-14, 1964.

[54] O. Supponen, D. Obreschkow, P. Kobel, M. Tinguely, N. Dorsaz, and M. Farhat. Shock waves from nonspherical cavitation bubbles. Journal of Fluid Mechanics, 2:093601, 2017. 
[55] S. Menon and M. Lal. On the dynamics and instability of bubbles formed during underwater explosions. Experimental Thermal and Fluids Science, 16:305-321, 1998. 